\title{
Evidence for the Emission of a Massive Neutrino in Nuclear Beta Decay
}

Eric B. Norman, Bhaskar Sur, K.T. Lesko, M.M. Hindi, Ruth-Mary Larimer i eressa R. Ho, Jon T. Wiltort, Paul N. Luke, William L. Hansen and Eugene E. Haller

\author{
Nuclear Science Division \\ Lawrence Berkeley Laboratory \\ University of California \\ Berkeley, Ca 94720, USA
}

This work was supported by the Director, Oflice of Energy Research, Division of Nuclear Physics of the Olfice of High Energy and Nuclear Physics of the U.S. Department of Energy under Contract DE-ACO3$76 S F 00098$ 


\section{Evidence for the Emission of a Massive Neutrino in Nuclear Beta Decay}

Eric B. Norman (1).(2), Bhaskar Sur (1).(2)*, K. T. Lesko (1),(2), M. M. Hindi (1),(3), RuthMary Larimer (1),(2), Teressa R. Ho (1), Jon T. Witort (2), Paul N. Luke (4), William L. Hansen (4), and Eugene E. Haller(4),(5)

(1) Nuclear Science Division, Lawrence Berkeley Laboratory, Berkeley, CA 94720

(2) Center for Particle Astrophysics, University of California, Berkeley, CA 94720

(3) Physics Department, Tennessee Technological University, Cookeville, TN 38505

(4) Engineering Division, Lawrence Berkeley Laboratory, Berkeley, CA 94720

(5) Dept. of Materials Science and Mineral Engineering, Univ. of California, Berkeley, CA 94720 , U.S.A.

\section{ABSTRACT}

We have studied the $\beta$-spectrum of ${ }^{14} \mathrm{C}$ using a germanium detector containing a crystal with ${ }^{14} \mathrm{C}$ dissolved in it. We find a feature in the $\beta$ spectrum $17 \mathrm{keV}$ below the endpoint which can be explained by the hypothesis that there is a heavy neutrino emitted in the $\beta$-decay of ${ }^{14} \mathrm{C}$ with a mass of $17 \pm 2 \mathrm{keV}$ and an emission probability of $1.40 \pm 0.45 \%$. In addition, we have studied the inner bremsstrahlung spectrum of $55 \mathrm{Fe}$ and also find indications of the emission of a $\sim 17-\mathrm{keV}$ neutrino. These results are consistent with observations of similar anomalies in the $\beta$-decays of ${ }^{3} \mathrm{H}$ and ${ }^{35} \mathrm{~S}$.

The existence of massive neutrinos would have profound implications for both particle physics and astrophysics. The observation of an anomaly, or "kink", $17 \mathrm{keV}$ below the end-point in the $\beta$-spectrum of ${ }^{3} \mathrm{H}$ was first reported in 1985 by Simpson [1] and interpreted by him to correspond to the emission of an electron antineutrino with a $3 \%$ admixture of a neutrino state of mass $=17 \mathrm{keV}$. This report was criticized [2-5] on grounds of both experimental method and data analysis. Moreover, it was quickly followed by a number of studies [6-13], all of which claimed to rule out the $3 \%, 17-\mathrm{keV}$ neutrino admixture hypothesis at various confidence levels. One of these data sets [7] was reanalyzed by Simpson [14] to show evidence for a $1-2 \%$ admixture of a 17-keV neutrino. Finally, in 1989, there appeared two reports of $\beta$-spectrum anomalies corresponding to a $\sim 1 \%$ admixture of a $17-\mathrm{keV}$ neutrino. Simpson and Hime[15] analyzed the $\beta$-spectrum of $35 \mathrm{~S}$. Hime and Simpson [16] studied the $\beta$-spectrum of ${ }^{3} \mathrm{H}$ implanted in a germanium detector. In the first of these papers, the authors also carefully criticized most of the previous null result experiments.

If Simpson's results are correct, then this "kink" should be present in all $\beta$ spectra. It is therefore important to test this claim for nuclei with different $Z$ and $A$. This would also provide a test of the possibility that the eflects observed by Simpson were due to some atomic physics phenomena peculiar to his choice of sources. These 
questions prompted us to mount experiments to look in detail at the $\beta$-spectrum of ${ }^{14} \mathrm{C}$ and the inner bremsstrahlung spectrum of ${ }^{55} \mathrm{Fe}$.

\section{B Spectrum of ${ }^{14} \mathrm{C}$}

The $\beta$ decay of ${ }^{14} \mathrm{C}$ is an allowed ground-state to ground-state transition with an endpoint energy near $156 \mathrm{keV}$. Moreover, we were aware of a unique detector produced by Haller et al. [17] that was ideally suited for this experiment. The detector contains a germanium crystal grown from a melt of germanium which had ${ }^{14} \mathrm{C}$ labelled carbon dissolved in it. This system thus functions as a windowless detector with a nearly ideal response function for the $\beta$ particles emitted by the ${ }^{14} \mathrm{C}$ inside the crystal. To produce such material, ${ }^{14} \mathrm{C}$-labelled methane was cracked into graphite on guartz crucibles. Germanium was then placed in these crucibles, melted, and pulled inte single crystals. Autoradiographs performed on the crystal used in the present study (Crystal No. 701 from Ret. 17) indicated that the ${ }^{14} \mathrm{C}$ is dispersed uniformly throughout the crystal. This detector has a ${ }^{14} \mathrm{C}$ concentration of $6 \times 10^{11} \mathrm{~cm}^{-3}$ and a planar $\mathrm{p}-\mathrm{i}-\mathrm{n}$ diode structure with a thickness of $1.28 \mathrm{~cm}$. The $\mathrm{n}+$ electrode is divided by a $1-\mathrm{mm}$ wide circular groove into a "center region" $3.2 \mathrm{~cm}$ in diameter and an outer "guard ring". By operating the guard ring in an anti-coincidence mode, one can reject events occurring near the boundary which are not fully contained within the center region. The ${ }^{14} \mathrm{C} \beta$ decay counting rate from the center region of the crystal is $20 \mathrm{~s}^{-1}$.

The present experiment was conducted at Lawrence Berkeley Laboratory's Low Background Counting Facility. A 1.3-cm thick brass plate was placed on the front face of the detector which was then placed inside a graded shield made of $\mathrm{Al}, \mathrm{Cu}, \mathrm{Cd}$, and Sn. Further shielding was provided by $10-15 \mathrm{~cm}$ of low activity lead surrounding the entire assembly. Signals from the center region and the guard ring portions of the ${ }^{14} \mathrm{C}$ crystai were separately processed through amplifiers using $4-\mu s$ shaping times. Signals from a two-channel precision pulser were fed through this detector at a rate of $5 \mathrm{~Hz}$ to monitor the gain and DC offset of the electronics. Data were taken using a PC-based acquisition system. Three separate spectra were accumulated from the detector: (1) center region, (2) center region in anti-coincidence with guard ring, and (3) guard ring. The guard ring veto signal used to gerierate spectrum (2) required that an event deposit more than $20 \mathrm{keV}$ but less than $183 \mathrm{keV}$ in the guard ring portion of the crystal. Data were collected in 4096 channels of $0.144 \mathrm{keV}$ width and were recorded in 1-day time bins on the magnetic disc of the computer.

The ${ }^{14} \mathrm{C}$ crystal was counted for a tolal of 122 days. After this counting period, the ${ }^{14} \mathrm{C}$ crystal was removed from the cryostat, and a similarly shaped carbon-free planar guard-ring germanium crystal was installed. Fifty-two days of background data were accumulated with this crystal. The centroids of the pulser peaks and those of the background gamma-ray lines showed no significant variation $(<0.1 \mathrm{keV})$ over the course of these data taking runs. Thus, all of the ${ }^{14} \mathrm{C}$ spectra were summed together and are shown ir Figure 1 (a). The result of summing all of the background spectra is shown in Figure 1(b). Using the ratios of the major $U$ and Th decay-chain $\gamma$-ray lines observed in the two spectra one can appropriately scale the background spectrum. The resulting background-subtracted ${ }^{14} \mathrm{C} \beta$ spectrum a total of $2.25 \times 10^{8}$ counts. 
There are $\sim 10^{6}$ counts in the last $17 \mathrm{keV}$ of this spectrum, and there are $\sim 10^{5}$ counts/keV at an energy of $139 \mathrm{keV}$ (i.e., $17 \mathrm{keV}$ below the endpoint).
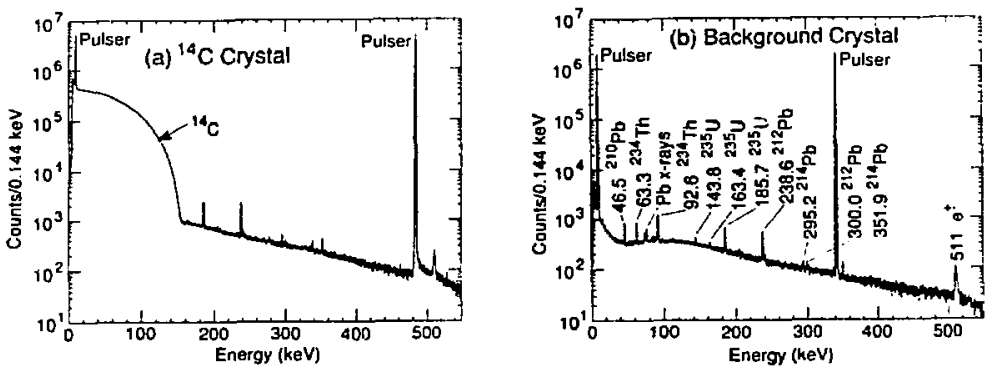

Fig. 1. (a) Spectrum observed from 122 days of counting with the ${ }^{14} \mathrm{C}$-doped germanium crystal. (b) Spectrum observed from 52 days of counting with the background crystal Gamma-ray energies are given in keV. Due to the different capacitances of the ${ }^{14} \mathrm{C}$-doped crystal and the background crystal, it was not possible to place the upper pulser peak in the same position in the two spectra.

If in nuclear beta decay, there are actually two decay channels open, one associated with $m_{v}=0$ and one with $m_{v} \neq 0$, then the spectrum of $\beta$ particles is given by the expression [18] :

$$
\frac{d N(E)}{d E}=(1-c) \frac{d N\left(E, m_{\nu}=0\right)}{d E}+c \frac{d N\left(E, m_{\nu}\right)}{d E},
$$

where

$$
\frac{d N\left(E, m_{\nu}\right)}{d E} \propto A F(Z, E) p E(W-E)\left[(W-E)^{2}-m_{\nu}^{2}\right]^{1 / 2} .
$$

In the case of ${ }^{14} \mathrm{C}$, the coefficient $\mathrm{c}$ is very nearly equal to the probability of heavy neutrino emission. $A$ is the overall spectrum normalization factor, $F(Z, E)$ is the Fermi function for $Z=7$ with the relativistic [19] and screening [20 ] corrections applied, $E_{e}$ and $p_{e}$ are the electron total energy and momentum, respectively, and $W$ is the total decay energy. There have been discussions over the years in the literature as to possible deviations in the shape of the ${ }^{14} \mathrm{C}$ beta spectrum from that expected for a pure allowed transition. $[21,22]$ To allow for possible smooth departures from an allowed shape, the above theoretical spectrum was multiplied by a "shape factor" of the form

$$
\left(1+\beta_{1}(W-E)+\beta_{2}(W-E)^{2}\right) \text {. }
$$

The resulting spectrum was then convoluted with the detector response function which we assume consists of a Gaussian shaped peak and a flat tail extendirig down to zero kinetic energy. The fraction of events in this tail is assumed to increase linearly with the $\beta$ energy. From measurements conducted with external gamma-ray sources and from the background lines observed during the data taking, we determined that the FWHM of the Gaussian peak is $1.0 \mathrm{keV}$ over the energy range of interest. From the 
known ranges and bremsstrahlung energy losses of $\beta$ 's in germanium, [23] we estimate that this tail contains at most $1.5 \%$ of all $156-\mathrm{keV} \beta$ - events originating in the center region of the crystal. The response function of this detector for electrons originating within it was also calculated using the Monte-Carlo code GEANT.[24] The results of these calculations indicate that this tail may actually contain only about $0.2 \%$ of all $\beta$ decay events. We therefore performed analyses with the tail set equal to 0 , $1.5 \%$ and $4 \%$ and obtained similar results.

The experimental data were then compared to the theoretically expected spectrum using a least-squares fitting procedure in which for given values of $m_{v}$ and $c$, the following five parameters were allowed to vary simultaneously: $A, W, \beta_{1}, \beta_{2}$, and the background normalization factor. This analysis was performed on the data in the energy range $100-160 \mathrm{keV}$ in both $0.144 \mathrm{keV}$ wide energy bins (418 data points) and on a data set compressed to $1 \mathrm{keV}$ per channel. The results of the analysis on the unbinned data are shown in Figure 2(a). The minimum value of $\chi^{2}$ obtained under the assumption of only massless neutrinos is 415 . This corresponds to the value of $\chi^{2}$ on both the horizontal (i.e. $m_{v}=0$ ) and on the vertical axis (i.e. $c=0$ ). The absolute minimum value of $\chi^{2}$ is 406 and is found for $m_{v}=17 \mathrm{keV}$ and $c=1.4 \%$. Thus, there is a difference of 9 units of $\chi^{2}$ between these two cases. This excludes the null hypothesis (i.e., no heavy neutrino emission) at the $99 \%$ confidence level. [25]
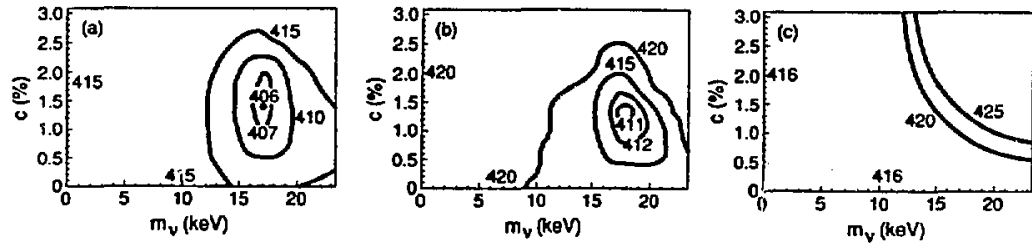

Fig. 2. Contour plots of $\chi^{2}$ as a function of the neutrino mass, $m_{v}$, and $c$ (where $c$ is defined by Eqn. 1). The curves are labelled by the values of $\chi^{2}$. (a) results from the analysis of our ${ }^{14} \mathrm{C}$ experimental data; (b) results from the analysis of Monte-Carlo generated data which contains a $1.4 \%$ fraction of a $17-\mathrm{keV}$ neutrino; (c) results from the analysis of Monte-Carlo generated data which contains only a zero mass neutrino.

To check on what sensitivity is expected from data of the quality we have obtained, we generated approximately 50 Monte-Carlo data sets corresponding to the case of (i) $m_{v}=17 \mathrm{keV}, c=1.4 \%$, and 5 data sets for the case (ii) $m_{v}=0$. These data sets were treated in exactly the same manner as the experimental data and were then analyzed using the least-squares method described above. Typical results for case (i) are shown in Fig. 2(b). The minimum value of $\chi^{2}$ obtained from this data set was 411 for $m_{v}=17.5 \mathrm{keV}, c=1.2 \%$. The value of $\chi^{2}$ found for $m_{v}=0$ in this data set was 420 . Figure 2(c) shows the results of the fitting procedure applied to an example of case (ii). The minimum value of $\chi^{2}=416$ occurs, as expected, for $m_{v}=0$, and the value 
obtained for $m_{v}=17 \mathrm{keV}, c=1.4 \%$ is 9 units larger. These results demonstrate that our experiment has the statistical sensitivity to distinguish between these two cases at the level observed in our experimental data.

Various projections of these results can be made by fixing one parameter and allowing all others to vary in such as way as to minimize $\chi^{2}$. The results of this procedure for projections of $m_{y}, c$, and the ${ }^{14} \mathrm{C} \beta$-endpoint energy, $E_{0}=\left(W-m_{e}\right)$, are shown in Figure 3. From these projections, we obtain $m_{v}=17 \pm 2 \mathrm{keV}, c=1.40 \pm$ $0.45 \%$ and $E_{0}=155.74 \pm 0.03 \mathrm{keV}$ ( all uncertainties are $1 \sigma$ ). The present result for the endpoint energy does not agree with the value of $156.476 \pm 0.005 \mathrm{keV}$ deduced from the mass spectrometry data of Smith and Wapstra, [26] but falls in the middle of the results of previous beta endpoint energy measurements. [26]
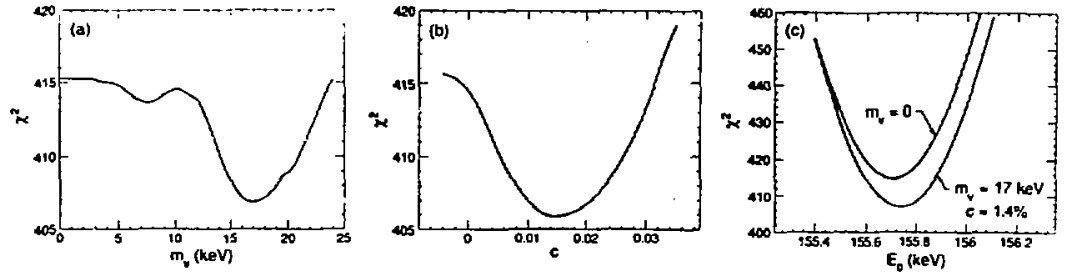

Fig. 3. (a) $\chi^{2}$ vs. the neutrino mass, $m_{v}$. (b) $\chi^{2}$ vs. c. (c) $\chi^{2}$ vs. the ${ }^{14} \mathrm{C}$ endpoint energy. Separate curves are shown for the cases of $m_{v}=17 \mathrm{keV}$, and $m_{v}=0$.

To illustrate the degree to which the calculated spectra agree with the data, we have divided the data by the results of the best fit obtained under the assumption of only massless neutrinos. This is illustrated in Fig. 4 (a) for our experimental data, and in Fig. $4(b)$ for Monte-Carlo data generated with $m_{v}=17 \mathrm{keV}$ and $c=1 \%$. For display purposes, the data were compressed into $1-\mathrm{keV}$ wide bins. The horizontal line is the expectation for massless neutrinos. The curve shown in part (a) is what one obtains by taking a spectrum containing a $1.4 \%$ admixture of $17 \mathrm{keV}$ neutrinos (i.e., the best fit to the experimental data) and dividing it by the best fit obtained for $i n_{v}=0$. The curve shown in part (b) is obtained by taking a spectrum containing a $1.2 \%$ admixture of 17.5 $\mathrm{keV}$ (i.e.the best fit to the Monte-Carlo data) and dividing it by the best fit obtained for $m_{v}=0$. While the difference in agreement between the data and the two fits is not striking to the eye, the statistical analysis indicates 9 units of $\chi^{2}$ difference between the two curves, most of which is generated in the vicinity of the "kink."

We have performed similar analyses on a smaller data set covering the energy range $125-160 \mathrm{keV}$ using both the experimental and Monte Carlo generated data. Using the values of $\beta_{1}$ and $\beta_{2}$ determined from the fits to the wider energy interval, the results of this analysis again show that a $\sim 1 \%$ emission probability of a $17 \mathrm{keV}$ neutrino gives a $\chi^{2} 9$ units lower than that obtained assuming only massless neutrinos. We have also pertormed tests to determine if some aspect of the detector response could account for the "kink." Using external $\gamma$-ray sources, we searched for an anomaly $17 \mathrm{keV}$ below the pholopeak and found no such feature. We did observe the $\mathrm{Ge}_{\theta} \mathrm{X}$-ray escape peak which occurs $10 \mathrm{keV}$ below the photopeak. For a 122-keV $\gamma$ 
ray, this peak is $0.1 \%$ as large as the photopeak and therefore cannot account for our result.

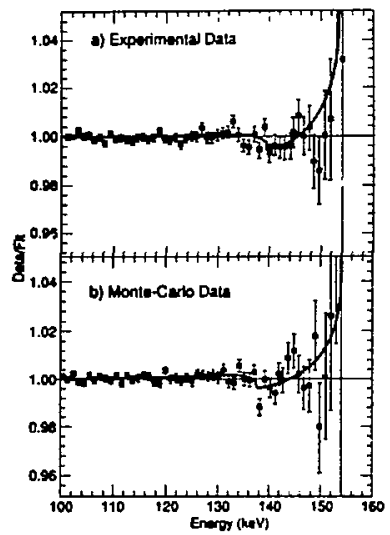

Fig. 4. The ratio of the ${ }^{14} \mathrm{C}$ data to a theoretical fit assuming the emission of only zero-mass neutrinos. The data were compressed to $1 \mathrm{keV} / \mathrm{channel}$. The horizontal line is the shape expected for zero-mass neutrinos. The curves illustrate the shape expected from the best fits to the data. (a) analysis of experimental data; (b) anaylsis of Monte-Carlo generated data which contain a $1 \%$ f: zction of a $17-\mathrm{keV}$ neutrino.

\section{Inner Bremsstrahlung Spectrum of $55 \mathrm{Fe}$}

The electron-capture decay of $55 \mathrm{Fe}$ is an allowed ground-state to ground-state transition with a QEC value of $231.7 \mathrm{keV}$ and the probability of radiative electron capture, or inner bremsstrahlung (IB), is $3.25 \times 10^{-5}[27]$ Although a previous search for the emission of massive neutrinos in this decay yielded a null result [13], we felt that a second experiment was warranted.

A $10 \mathrm{mCi}$ source of $55 \mathrm{Fe}$ (containing a small amount of $59 \mathrm{Fe}$ ) was chemically purified and the resulting $\sim 2 \mathrm{~cm}^{3}$ of liquid was contained in a glass vial. The source was carefully positioned against the front face of a $1.3-\mathrm{cm}$ thick plariar germanium detector. As shown in Fig. 5, this entire assembly was then placed inside a Nal anticoincidence shield consisting of a $30-\mathrm{cm}$ by $30-\mathrm{cm}$ annular detector and a $7.5-\mathrm{cm}$ by $15-\mathrm{cm}$ detector. These $\mathrm{Nal}$ cietectors vetoed both Compton-scattered IB photons as well as external background radialion. The ${ }^{55} \mathrm{Fe}$ IB counting rate in the germanium detecior was approximately $1000 \mathrm{~s}^{-1}$.

Data were taken using the same PC-based aquisition system as was used in the ${ }^{14} \mathrm{C}$ experiment. The $55 \mathrm{Fe}$ source was counted in 1-day time bins and the data were recorded on the disc of the computer. At the end of each day, the source was removed and room background measurements were conducted for several hours. Finaliy, energy calibration measurements were periormed. This procedure was repeated 20 times. Using a number of different liquid sources placed in the same 
geometry as the $55 \mathrm{Fe}$ source, we measured the detector resolution, efficiency, and photopeak/Compton ratio as a function of $\boldsymbol{\gamma}$-ray energy.

P.M.T.'s

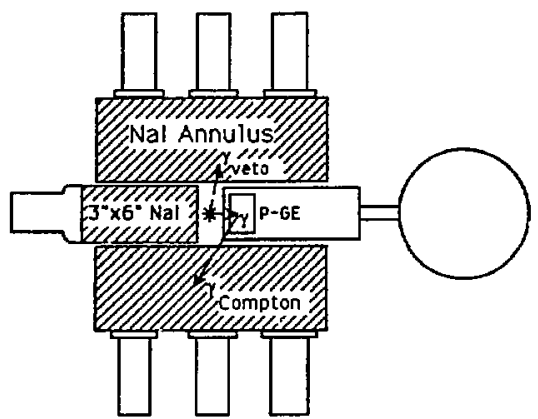

Fig.5. Schematic view of the detectors used in the $55 \mathrm{Fe}$ inner bremsstrahlung experiment.

The result of summing together the 20 days worth of $55 \mathrm{Fe}$ data is shown in Fig. 6. From this spectrum, we subtracted the ${ }^{59} \mathrm{Fe}$ contribution, room background, and pileup events. The resulting net ${ }^{55} \mathrm{Fe}$ IB spectrum contains $\sim 10^{9}$ total counts. There are again $\sim 10^{6}$ counts in the last $17 \mathrm{keV}$ of this spectrum and $\sim 10^{5}$ counts/keV 17 keV below the endpoint.

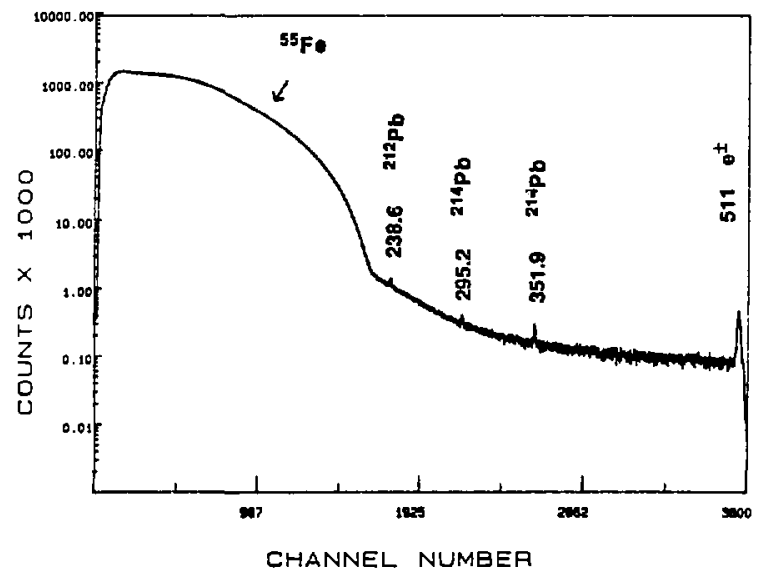

Fig. 6. Inner bremsstrahlung spectrum of $55 \mathrm{Fe}$. Background gamma-ray energies are given in $\mathrm{keV}$. 
The observed ${ }^{55} \mathrm{Fe}$ IB spectrum was compared to the expected shape using a least-squares fitting procedure analogous to that employed in the analysis of the ${ }^{14} \mathrm{C}$ data. Theoretical IB spectra for electron capture from the $1 \mathrm{~s}, 2 \mathrm{~s}, 2 \mathrm{p}$, and $3 \mathrm{~s}$ shells were calculated using the methods of Bambynek ot al [28]. These spectra were then convoluted with a detector response function inferred from the source measurements described above.

The results of this litting procedure applied to the last $50 \mathrm{keV}$ of the $55 \mathrm{Fe}$ IB spectrum are illustrated in Fig. 7. The best fit to this data set, which has 497 degrees of freedom, yields $m_{v}=21 \pm 2 \mathrm{keV}$ and $c=(0.85 \pm 0.45$ ) \% (all uncertainties are $1 \sigma$ ). These results indicate that there is a "kink" in the $55 \mathrm{Fe}$ IB spectrum, but corresponding to a slightly different $m_{v}$ value than that found in the study of ${ }^{14} \mathrm{C}$ decay. Also, the overall $x^{2}$ values obtained here are not as good as those found in the analysis of the ${ }^{14} \mathrm{C}$ data. We believe both of these effects are due to our lack of precise knowledge of the detector response function. Fits performed in which we varied the energy dependence of the detector efficiency showed that the position of the "kink" could be moved. Thus, these results are suggestive that there is a feature $\sim 17 \mathrm{keV}$ below the endpoint of the $55 \mathrm{Fe}$ IB spectrum, but further study of this system is clearly necessary.
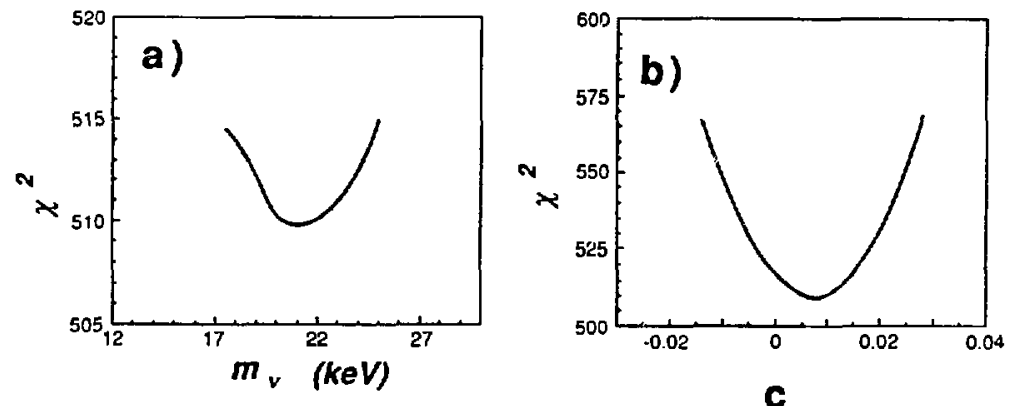

Fig. 7. Results of least-squares fits to the ${ }^{55} \mathrm{Fe}$ inner bremsstrahlung data. (a) $\chi^{2}$ vs. the neutrino mass, $\mathrm{in}_{v}$. (b) $\chi^{2}$ vs. c.

\section{Conciusions}

The results of the present studies of the $\beta$ spectrum of ${ }^{14} \mathrm{C}$ and the inner bremsstrahlung spectrum of ${ }^{55} \mathrm{Fe}$, thus support the claim by Simpson that there is a $17-\mathrm{keV}$ antineutrino emitted with $-1 \%$ probability in nuclear beta decay. The analyses of our ${ }^{14} \mathrm{C}$ data rule out the null hypothesis (i.e., no heavy neutrino emission) at the $99 \%$ confidence level. These findings are in agreement with similar positive results obtained in a study of the inner bremsstrahlung spectrum of ${ }^{71} \mathrm{Ge}[29]$. We intend to continue our studies of ${ }^{14} \mathrm{C}$ with an improved detector that will contain a crystal with a much higher amount of ${ }^{14} \mathrm{C}$. This will provide much greater sensitivity to the presence of massive neutrinos. 


\section{Acknowledgements}

We wish to thank C. P. Cork, F. S. Goulding, D. A. Landis, A. R. Smith, and K. L. Wedding for their assistance in various aspects of this work. We also wish to thank E. D. Commins and L. A. Khalfin for their comments and suggestions regarding this manuscript. This work was supported in part by the Nuclear Physics Division of the U. S. Department of Energy under Contract No. DE-AC03-76SF00098, and in part by the Center for Particle Astrophysics, a U. S. National Science Foundation Science and Technology Center operated by the Univ. of California at Berkeley under Cooperative Agreement No. AST-8809616. M. M. Hindi is also supported in part by the U. S. Department of Energy under Contract No. DE-FG05-87ER40314.

\section{References}

- Present address: Physics Department, Queen's University, Kingston, ON, Canada

[1] J. J. Simpson, Phys. Rev. Lett. 54, 1891 (1985).

[2] B. Eman and D. Tadic, Phys. Rev. C 33, 2128 (1986).

[3] J. Lindhard and P. G. Hansen, Phys. Rev. Lett. 57, 965 (1986).

[4] W. C. Haxton, Phys. Rev. Lett. 55, 807 (1985).

[5] G. R. Kalbfleisch and K. A. Milton, Phys. Rev. Lett. 55, 2225 (1985).

[6] T. Altzitzoglou et al., Phys. Rev. Lett. 55, 799 (1985).

[7] T. Ohi et al Phys. Lett. 160B, 322 (1985).

[8] V. M. Datar et al., Nature (London) 318, 547 (1985).

[9] A. Apalikov et al., Pis'ma Zh. Eksp. Teor. Fiz. 42, 233 (1985)

[10] J. Markey and F. Boehm, Phys. Rev. C 32, 2215 (1985).

[11] D. W. Hetherington et al., Phys. Rev. C 36, 1504 (1987).

[12] M. J. G. Borge et al., Physica Scripta 34, 591 (1986).

[13] I. Zlimen, S. Kaucic, A. Ljubicic, and B. A. Logan, Physica Scripta 38, 539 (1988).

[14] J. J. Simpson, Phys. Lett. 174B, 113 (1986).

[15] J. J. Simpson and A. Hime, Phys. Rev. D 39, 1825 (1989).

[16] A. Hime and J. J. Simpson, Phys. Rev. D 39, 1837 (1989).

[17] E. E. Haller, W. L. Hansen, P. Luke, R. McMurray, and B. Jarrett, IEEE Traris. Nucl. Sci. Vol. NS-29, No. 1, 745 (1982).

[18] F. Boehm and P. Vogel, Physics of Massive Neutrinos, (Cambridge Univ. Press, Cambridge, 1987) p. 68.

[19] H. Behrens and J. Janecke, Landolt-Bornstein New Series (Springer, Berlin, 1969), Group I, Vol. 4.

[20] M. E. Rose, Phys. Rev. 49, 727 (1936).

[21] A. V. Poinm, R. C. Waddell, J. P. Powers, and E. N. Jensen, Phys. Rev. 97, 432 (1955).

[22] Ch. Sonntag et al., Lett. Nuovo Cim. IV, 717 (1970).

[23] L. Pages, E. Bertel, H. Joffre, and L. Sklavenitis, Atomic Data 4, 1 (1972).

[24] R. Brun, F. Bruyant, M. Maire, A. C. MiPherson, and P. Zanarini, GEANT3, CERN, DD/EE/84-1 (1987).

[25] W. H. Press, B. P. Flannery, S. A. Teukolsky, and W. T. Vetterling, Numerical Recipes (Cambridge Univ. Press, Cambridge, 1986) p. 536.

[26] L. G. Smith and A. H. Wapstra, Phys. Rev. C 11,1392 (1975). 
[27] E. Browne and R. B. Firestone, Table of Radioactive Jsotopes (John Wiley \& Sons, Now York, 1986).

[28] W. Bambynek et al., Rev. Mod. Phys. 49, 77 (1977).

[29] I. Zlimen, A. Ljubicic, S. Kaucic, and B. A. Logan, Proc. 14th Europhys. Conf. on Nucl. Phys., Bratislava, Czechoslovakia, Oct. 1990. 